\title{
Post Caesarean Vesicouterine Fistula Causing Menouria
}

\author{
Sultana $\mathrm{R}^{1}$, Haque $\mathrm{N}^{2}$
}

\begin{abstract}
Vesicouterine fistula is an uncommon urogenital fistula. The incidence is on the rise because of increasing incidence of Caesarean sections. Cyclical Haematuria or Menouria is an important clinical feature of this fistula which may or may not be associated with urinary incontinence depending on the location of the fistulous tract. We present a case report of Post caesarean section Vesicouterine fistula following 2 Caesarean sections. This was successfully managed by laparotomy with repair of fistulous tract in bladder wall and Total Abdominal Hysterectomy for multiple Fibroid uterus done. Menouria is a rare event in Gynecology and one should always keep this possibility in mind when there is cyclical haematuria. A 42 year old Bangladeshi woman was hospitalized with complaints of menorrhagia, lower abdominal pain for last 8 years, cyclical hematuria for last 20 years. She was mildly anemic, haemodynamically stable and regularly menstruating women. The primary Ultrasound scans suggested multiple fibroid with cystic ovary in left side. Cystoscopy was done and findings are a fistulous opening in the bladder measuring around $7 \mathrm{~mm}$ in size. It was supratrigonal in position. The patient had no history of Endometriosis, Pelvic irradiation therapy, Inflammatory disease, Trauma or Malignancy. Initially there was dilemma in her diagnosis and the patient was diagnosed as a case of bladder Endometriosis besides fibroid uterus. So surgery was planned and Total Abdominal Hysterectomy \& bilateral salphingo-oophorectomy done. There was a fistula about $3 \mathrm{~cm} \times 2 \mathrm{~cm}$ in the lower part of the body of uterus connecting with the base of Bladder. Fistula repaired after dissection, patient follow up done and catheter removed after 14 days without any complications. Vesicouterine fistula can be prevented if care is taken to separate the bladder from the uterus during repeat Caesarean sections.
\end{abstract}

\section{Introduction}

Vesicouterine fistula (VUF) is a rare type of fistula accounting for only $1-4 \%$ of all cases of Urogenital fistula. However the incidence of VUF has been on the rise due to increasing incidence of Lower Uterine Cesarean Section. It is seen more often after repeat cesarean section rather than after the primary ${ }^{1-2}$.Menouria is a term coined for vesical menstruation ${ }^{3}$. It follows a fistulous defect above the level

1. Dr. Razia Sultana DGO, FCPS

Senior Consultant, Department of Gynae

Gazi Medical College and Hospital, Khulna

2. Corresponding Authors: Dr. Nazmul Haque FCPS, MS Consultant Urology

Shaid Shiekh Abu Naser Specialized Hospital, Khulna of internal os, communicating with the bladder. This results in cyclical haematuria and apparent amenorrhea in an otherwise continenent lady. This clinical picture is also known as Youssef,s syndrome. Endometriosis of the Bladder is differential diagnosis for Menouria. VUF initially thought to be a complication of Lower Segment Caesarean Section, a variety of causes are identified including congenital, post biopsy complication, irradiation and even following the placement of Intrauterine contraceptive device $^{3-10}$. Since it is an uncommon condition, a variety of preoperative imaging methods have been employed for its evaluation and planning treatment. These include Ultrasound (US), Cystography,CT scan, Cystoscopy, Hysterography and $\mathrm{MRI}^{7,8,10,12}$ with varying results. X-ray techniques which are easy to conduct and provide reproductive images of the anatomy of the genitourinary tract.VUF following Caesarean section may heal spontaneously with involution of the uterus. Spontaneous healing may occur in 5\% of cases, when it does not occur, continuous hormonal therapy can be given to suppress menstruation for 3-6 months as first line of therapy.Electrocoagulation of the fistula can be tried in cases of small fistula.

\section{Case Report}

A 45 year old lady came with $\mathrm{H} / \mathrm{O}$ cyclical haematuria for last 20 years. SheWas married for 25 years, have 3 issues, all were caesarean delivery. She was menstruating. There was no H/O Incontinence normally. She was not using any contraceptive. Examination revealed average build of the patient. A longitudinal thick scar was present in the abdomen. No mass was palpable. Per vaginal exam showed vulva, vagina and urethral meatus normal. Per speculum examination revealed cervix mildly congested, Uterus was about 12 week's size, both fornices were free. In anterior fornix there is attachment of bladder with the anterior wall of the cervix. No fistula felt in the vagina. Sonography revealed multiple fibroid with left sided ovarian cyst, Right ovary normal and other abdominal organs were normal. Here renal function tests were within normal reference range. Cystoscopy findings were there was fistula in the posterior wall of the bladder, supratriigonal in position and blood is coming out from the uterus. A provisional diagnosis of Bladder Endometriosis was made, Surgery was planned and laparotomy performed through lower transverse incision.

Uterus having multiple fibroid and there was fistula in between the base of bladder and cervix, Separation of fistula done by dissection and bladder fistula repaired in two layers and Total Abdominal Hysterectomy and Bilateral 
Salphingo-oophorectomy done and catheter left for 14 days. Therefore case was diagnosed as vesicouterine fistula. The patient made a steady recovery and was discharged on 7 th postoperative day and catheterwas removed after 14 days.

\section{Discussion}

Vesicouterine fistula are uncommon and pathological communication between the bladder and the uterus or the cervix $^{1}$ and represents $1-4 \%$ of the Urogenital fistulas with a peak incidence in young women between $25 \& 33$ years old ${ }^{4,7,8}$. The exact epidemiology is not well known. The rise in prevalence may be explained by an increase in Caesarean section and increase in vaginal delivery following Caesarean section. The main cause of urogenital fistula can be classified as Obstetrical, Surgical, Radiation, Necrosis or related to Malignancy. Currently the main cause of VUF is an iatrogenic injury during Caesarean section which accounts for $83-88 \%$ cases $^{4,7}$. These injuries occur two times more often after repeat Caesarean section ${ }^{7,8}$. Some risk factors have been advocated for the development of VUF, such as an inadequate reflection of the bladder wall from lower uterine segment, excessive intraoperative bleeding, severe dystocia, Forceps delivery, Manual removal of the placenta, Placenta percrata, Uterine rupture, previous Caesarean section and Dilatation \& Curettage. Other less frequent causes are Inflammatory Bowel diseases Endometriosis Intrauterine device migration, Bladder Tuberculosis and Congenital lesions ${ }^{2-9}$. Repeat Caesarean section may result in progressive devitalisation and scarring of the uterus and bladder base by damaging their vascular network thus predisposing to fistula formation ${ }^{4,7}$. Patients with vesicouterine fistula can have various clinical presentations. Usually immediate features occur when there is direct injury to the bladder during surgery. Patients can have early haematuria and/or urinary leakage, voiding difficulty, low grade pyrexia, urinary sepsis or complete asymptomatic ${ }^{9}$. Delayed presentation can occur when there is infection or a progressive devitalisation of the posterior wall of the bladder ${ }^{4,8}$. And patient often have symptoms of urinary leakage from the vagina, if the Cervix is incompetent, cyclic haematuria (menouria), Amenorrhea, Infertility or first trimester Abortion ${ }^{4,8}$.

Accurate and early diagnosis of VUF can be difficult. There are multiple means of investigating for VUF and several examinations may be required to confirm the diagnosis. The main stay of diagnosis is Cystoscopy and urinary tract imaging $^{2}$. Additional diagnostic procedures include contrast enhanced CT, MRI procedures.

Treatment options for VUF include conservative, medical or surgical treatment ${ }^{4,8,11,12}$. Some studies advocate conservative treatment for early diagnosed small fistula with bladder catheterization alone with antibiotics for 3 weeks ${ }^{2}$.Other proposed, induced amenorrhea by Oral contraceptive pill or Luteinizing Hormone Releasing Hormone analogs ${ }^{4}$. There are some reports describing the endoscopic fulguration of the fistula in up to $5 \%$ cases may be expected with the involution of the uterus. Surgery is the treatment of choice in most cases, especially for large VUF which invariably needs surgical closure ${ }^{4,10}$ Surgical repair of VUF are performed by different approaches, which include, Vaginal, Transvesical, Transperitoneal and Laparoscopic procedures ${ }^{11}$.

Vesicouterine fistula with vaginal urinary leakage, cyclic hematuria (menuria), amenorrhea Infertility and first trimester abortions. The diagnosis is ruled out by showing the fistulous tract between the bladder and uterus as well as by excluding other more frequent urogenital fistulas. The cases were diagnosed on the basis of medical history, radiological examinations and cystocopy. Thevesicouterine fistulas are often secondary to Caesarean section or abnormal delivery, the treatment is surgical but is above all preventive by the improvement of obstetric taking care and avoiding the vesical injuries in the course of Caesarean section.

\section{References}

1. Jozwtik M, Jozwick M, Lotocki W, Vesouterine fistulaan analysis of 24 cases from Poland-Int J GynecolObstet, 1997;57:169-72.

2. Hadzi-Djokic JB, Pejcic TP, Colovic VC-Vesicouterinefistula report of 14 cases BJU Int 2007;100:1361-3.

3. Youseff AF, Menouria following Lower uterine segment caesarean section Am J ObstetGynecol 1957;73:759-67.

4. Leukovsky-Z, Pode D Shaprio A-Vesicouterine fistula: a rare complication of Caesarean section. J Urol 1988;139:123-5.

5. Trauser ML.Vesicouterine fistula-a review Obstet Gynecol Survey 1986;4:743-53.

6. Thanos N, Pavlakis AJ, Davillas N. Vesicouterine fistula Urology 1986;28:426-8.

7. Raunch RJ Rodgers, MW. Spontaneous closure of Vesicouterinefistula following Caesarean section, JAMA 1962;181:997-9.

8. Rubino SM.Vesicouterine fistula treated amenorrhoea induced with contraceptive steroids: two case reports. Br Jr ObstetGynecol 1980;87:343-4.

9. Nercedar VP, McGuckju JF Jr, Caroline DF Chatwani A, Seidmon A. CT of vesicouterine fistula with Menouria:a complication of uterine biopsy, Jcompt Assist Tomogr 1995;19:324-5.

10. Schwaritzworld D, Mooppan UM, Taucer ML, Lean Kim. Vesicouterine fistula with menouria; a complication from an intrauterine contraceptive device J Urol 1986;136:1066-7.

11. Verthoven AT. Primary menouria due to congenital vesicouterine fistula ObstetGynecol. 1999;41:515-20.

12. Biomel V, Rofstad EK, Tropec, Lien HN, Critical soft tissue of the feamale pelvis: serial MR imaging before, during and after radiation therapy. Radiology 1997;20:391-7.

13. DiMarco CS, DiMaro DS, Klingele CI, Vesicouterine 
fistula: a review of eight cases. IntUrogynecol J pelvic floor Dysfunction 2006;17:395-0.

14. Yokoyana M, ArisamuC, Ando M. Successful management of Vesicouterine fistula by Leutinising hormone releasing hormone analog. Int J Urol 2006;13: 467-9.

15. Parecare AB, Zicar M, Zectini Anteniol S, Vesicouterine fistulas followng Caesarean section: report on a case, review and update of the literatureIntUrolNephrol 2002;34:335-44.

16. Al Nauatn KattanS Mustafa MS. Vesicouterine fistula after a previos low vertical caesarean section. Int J GynecolObstet 1996;55:161-2.

17. Navi JM, Rose M, Shaunik A, Conservative management of Vesicouterine fistula after uterine rupture. IntUrogynecol J, Pelvic floor dysfunction 2004; 15:434-5.
18. Jaswick M, Jazwiklotocki W. Vesicouterine fistula- an analysis of 24 cases from Polant. Int J GynecolObstet 1998;57:169-72.

19. Sefriovio, BenabbeTaarj H, Azyez M, Vesicouterine fistula of obstetrical origin. Report of 3 cases Am Urol (Paris) 2002;36:376-80.

20. Alkatib M, Franko AV, Fynes MM. Vesicouterine fistula following Caesarean delivery-Ultrasound diagnosis and surgical managemment. Ultrasound ObstetGynecol 2005;26:183-5.

21. Tahan F, Erbay E, Penbegil N, etal. Minimal invasive tretment of Vesicouterine fistula: a case reportIntUrolNephrol 2007;39:791-3.

22. Jozwik M, Jaswik M. Spontaneous closure of Vesicouterine fistula. Account for effective hormonal treatment. UrolInt 1999;62:183-7. 